\title{
Development of an MRI-Compatible Device for Prostate Focal Therapy
}

\author{
Jeremy Cepek ${ }^{1}$, Blaine Chronik $^{1}$, Uri Lindner ${ }^{2}$, \\ John Trachtenberg ${ }^{2}$, and Aaron Fenster ${ }^{1}$ \\ ${ }^{1}$ Robarts Research Institute, Western University, London, Canada \\ \{jcepek, afenster\} arobarts.ca, bchronik@uwo.ca \\ ${ }^{2}$ Department of Surgical Oncology, Division of Urology, \\ University Health Network, Toronto, Canada \\ lindneruri@gmail.com, john.trachtenberg@uhn.ca
}

\begin{abstract}
We present a device that has been developed for delivering prostate focal thermal therapy under magnetic resonance imaging (MRI) guidance. Unlike most existing devices, ours is capable of delivering needles to targets in the prostate without removing the patient from the scanner. This feature greatly reduces procedure time and increases accuracy. The device consists of a mechanical linkage encoded with optical incremental encoders, and is manually actuated. A custom magnetic resonance (MR) compatible alignment interface allows the user to manually align the device to its target with high accuracy inbore in very short time. The use of manual actuation over motors greatly reduces the complexity and bulk of the system, making it much more compact and portable. This is important when dealing with such tight space constraints. Needle targeting experiments in gel phantoms have demonstrated the device's ability to deliver needles with an accuracy of $2.1+/-1.3 \mathrm{~mm}$.
\end{abstract}

Keywords: MRI-compatible device, prostate cancer, focal thermal therapy.

\section{Introduction}

\subsection{Background}

It is estimated that over 240,000 men will be diagnosed with prostate cancer (PCa) in the United States in 2012, and over 28,000 men will die from it [1]. While treatments of the disease can be very effective if caught in the early stages, there is a growing belief among many clinicians that PCa is being over-treated with radical approaches. As a result, patients are being subjected to unnecessary morbidity, particularly those with low-risk disease. For patients in the low-risk category, disease management options range from radical prostatectomy to active surveillance (AS). AS has proven to reduce over-treatment [2], but the dropout rate is about $20 \%$, as the disease either progresses or the patient makes the decision to request definitive therapy [3]. In some of these cases complete radical resection may be "therapeutic overkill", and leave the patient with reduced sexual, urinary and bowel functions. Alternatively, properly 
executed selective focal ablative therapy could provide an effective option for definitive disease management with minimal treatment-related morbidity. Thus, there is a need for an accurate method of delivering focal prostate therapies.

One of the most common methods of delivering needles to the prostate for biopsy or therapy is with the aid of transrectal ultrasound (TRUS) imaging. While TRUS does provide real-time visualization of needles during insertion, it is not capable of accurately locating tumours or clearly visualizing the peripheral zone; the site of $\sim 80 \%$ of prostate cancers [4]. Magnetic resonance imaging (MRI) has the ability to image tumours, delineate the peripheral zone, and display tissue temperature for thermal ablative procedures [4]. MRI is therefore a very promising imaging modality for guiding focal thermal ablation therapies, since it can provide accurate target localization, real-time needle trajectory and depth tracking, and monitoring of the ablative procedure, all in the same session.

\subsection{Previous Work}

Various authors have reported the development of devices for the delivery of needles to the prostate under MRI guidance [5,6,7,8]. Most of these devices use either pneumatic or ultrasonic motors to align a needle with a target, and require removal of the patient from the scanner for manual needle insertion. Verification of needle placement is then made by placing the patient back into the scanner and re-imaging [5,6]. This approach increases procedure time considerably, and reduces the potential needle placement accuracy due to increased patient motion. Other authors report fully automated devices [7,8]. With these devices, needle insertion is motorized, and patient safety is compromised, since there is no haptic feedback provided to the physician. In an attempt to overcome these issues, we have developed an MRI-compatible needle trajectory alignment device that is manually actuated, and allows for adjustment of the needle trajectory and manual needle insertion with the patient in-bore. It is hypothesized that this system will allow for accurate delivery of prostate focal thermal therapy with minimal procedure time, while maintaining patient safety. The target accuracy to be achieved is $+/-2.5 \mathrm{~mm}$, based on the finding that tumours of clinically significant size are at least $\sim 10 \mathrm{~mm}$ in diameter [9].

\section{Methods and Materials}

\subsection{Device Design}

We present a device that allows a physician to precisely align a needle guide with an intended target in the prostate under MRI guidance. This is achieved through the use of four linear motion stages attached to an arm that holds needle templates. Movement of the arm is achieved manually by the physician. Two locking handles allow the needle insertion point and trajectory to be independently adjusted using the alignment handle. This approach removes the complexity and bulk that would be added by using motors for positioning, and still allows accurate targeting in less than $40 \mathrm{~s}$ with the patient in-bore. The two rear templates allow the physician to insert needles 
without moving the patient out of the bore of the scanner by extending the point of needle insertion within reach. The vertical slides are supported by spring counterbalances and ensure the device's position is maintained when the handle is released. An MR-visible fiducial arrangement is used to register the device's initial position in the scanner, and subsequent movements are tracked with optical linear encoders. The device is shown in Figure 1.

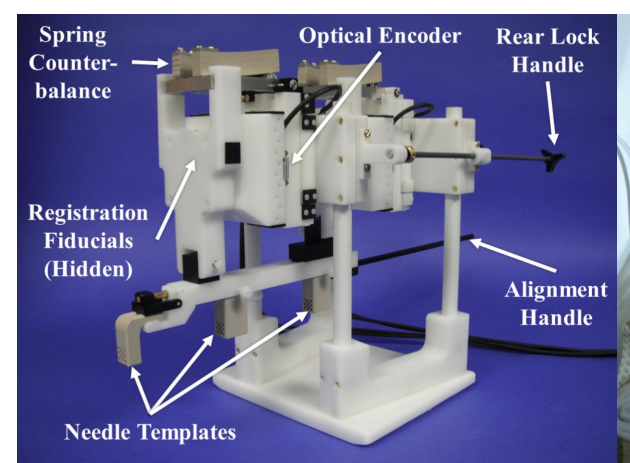

a)

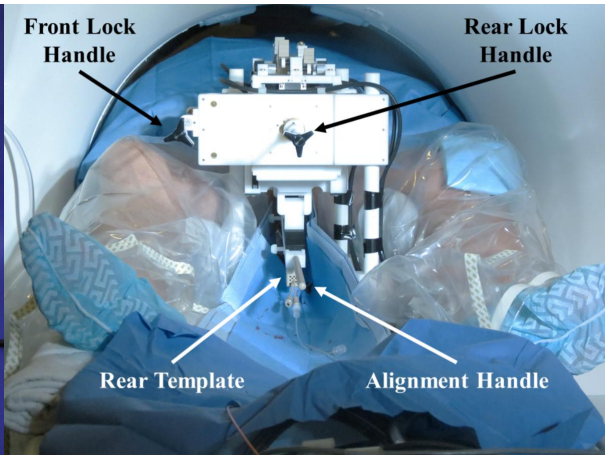

b)

Fig. 1. a) Device components, b) device in position with a patient

The patient is positioned head first supine in the scanner, and needles are inserted through the perineum. The needle is imaged as it is inserted, providing direct comparison between its position and the patient's anatomy. The templates allow for large tumours to be treated with multiple needles simultaneously.

User Interface. MR images are transferred from the scanner to a custom graphical user interface on a laptop via FTP. This interface allows the user to localize the device's registration fiducials and targets (tumours). The registration and target data is sent to a custom embedded electronics system that decodes linear position from the encoders and computes kinematics solutions. The kinematics solutions are used to send data to a custom MR-compatible visual alignment interface, placed immediately outside of the MRI bore. The interface indicates which direction the handle must be moved to reach the given target. Once the device has been aligned to the target within a given level of precision, only the middle LED in the grid will be displayed. Figure 2 shows this interface.

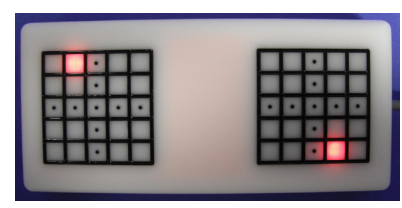

a)

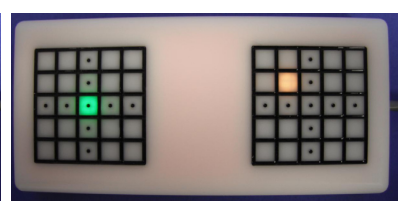

b)

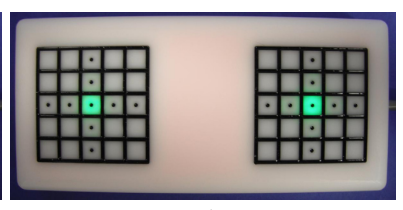

c)

Fig. 2. Alignment interface: a) not aligned, b) front stages aligned, rear stages within $3 \mathrm{~mm}$ in both directions, c) all stages within $0.25 \mathrm{~mm}$ of target 
Materials \& Components. All major components of the device are constructed of either Delrin or fiberglass. The linear ball slide bearings in the linear stages consist of stainless steel (grade 303) rods and titanium races with ceramic balls. All fasteners are either brass or plastic. Aluminum was used for various small components. Linear position encoding is achieved with non-magnetic incremental encoders (LIA-20, Numerik Jena, Jena, Germany). The encoders output a sine-cosine signal in the $\mathrm{kHz}$ range and do not interfere with the MRI's RF system. The encoders are connected to a custom embedded electronics interface inside the console room via RF filters in the scanner room's penetration panel.

Sterilization. The only components of the device that require full sterilization are the three needle templates. The templates are made from PEEK, which is autoclavable. The rest of the device is completely enclosed in a sterile plastic bag. The templates are attached to device through the plastic bag.

\section{$3 \quad$ Experimental Methods and Results}

\subsection{MR Compatibility}

It is important for the device to not interfere with the scanner's ability to produce high-quality images, and for the scanner to not interfere with the device's ability to accurately target tumours. Specifically, the presence of the device must not produce any distortion or reduction in signal-to-noise ratio (SNR) in the images, and the scanner must not interfere with the device's operation by introducing significant force, torque, vibration, or heating, or affect the operation of the encoders.

The distortion and SNR tests were based on the relevant ASTM and NEMA standards, respectively. Accordingly, these effects were evaluated by imaging a grid phantom located superior to the device for each of the following cases: 1 ) device not present (baseline), 2) device in position, not connected, 3) device in position, cables connected, not powered, 4) device in position, cables connected, powered. Since the device will not be moved during imaging, this case was not considered. The experimental setup is shown in Figure 3.

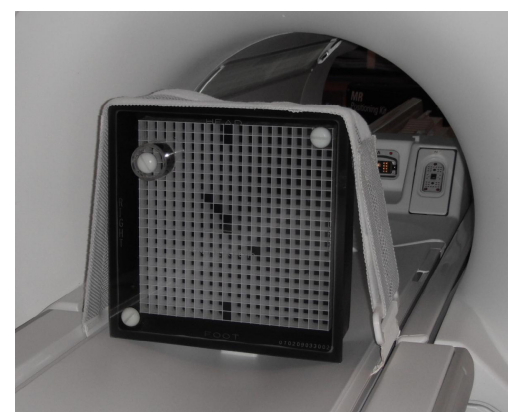

a)

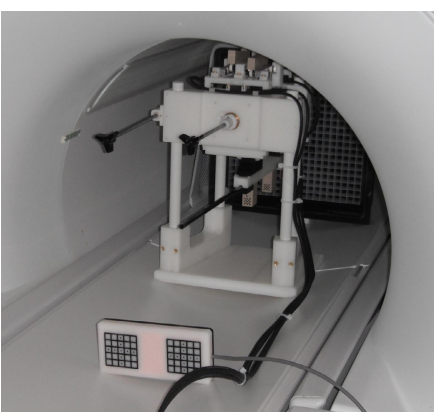

b)

Fig. 3. Setup for MR-compatibility tests: a) the grid phantom used, b) device positioning 
The device was positioned so the distance from the template to the center of the phantom was equivalent to the average distance to the center of a patient's prostate $(\sim 10 \mathrm{~cm})$, with the center of the phantom at the isocenter. Axial gradient echo images were acquired on a $3 \mathrm{~T}$ scanner (MR750, GE Healthcare, Milwaukee, WI) with the parameters: field of view (FOV): $400 \mathrm{~mm}$ x $400 \mathrm{~mm}$, matrix: 256 x 256, TR $=270$ $\mathrm{ms}, \mathrm{TE}=4 \mathrm{~ms}$, flip angle: $25^{\circ}$. Each set of images was acquired with bandwidths of both 195 and $977 \mathrm{~Hz} /$ pixel. The low bandwidth image set was used to evaluate image distortion, while the higher bandwidth set was used for SNR calculations. This presents an overestimate of the effect of the device on the images. There was negligible distortion in all cases. SNR was calculated using a mean signal in a 30 x 60 voxel region near the middle of the FOV, and the standard deviation of the signal in a region of the same size outside the phantom. The SNR was calculated in image slices that contained only fluid. SNR was found to decrease by no more than $6 \%$ from its value in case 1 in all other cases. MR thermometry images have also been acquired with the device present, and no significant interference was detected.

Effects of the scanner on the device were minimized by using non-magnetic materials for all major components. Non-magnetic metals were used only for small components, and did not cause any significant force, torque, heating or vibration effects. Fidelity of the encoder operation is ensured by rechecking that the encoder counts are zero each time an index pulse is received.

\subsection{Calibration}

Open-Air Calibration. An experiment was performed to quantify the device's ability to place a needle at a target in open air. The experiment involved fitting the manipulator arm with two steel tooling balls, and measuring their positions over the device's full range of motion using a coordinate measuring machine (CMM). The CMM has a total volumetric accuracy of $5.2 \mu \mathrm{m}$. Error in position of the furthest tooling ball (representative of the placement of a needle tip at the base of the prostate) was found to be $0.29+/-0.11 \mathrm{~mm}$.

MRI Calibration. Calibration of the device within the MRI workspace requires careful consideration of the sources of geometric errors present in MR images. Figure 4 shows a breakdown of the major sources of error that contribute to the total error in needle targeting.

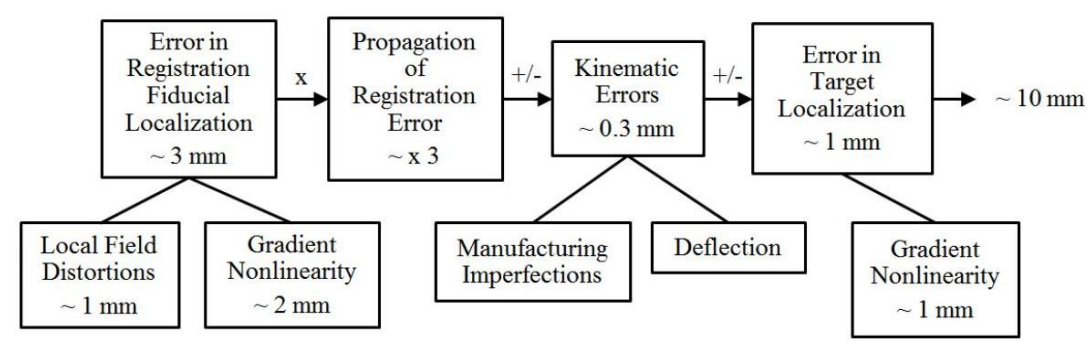

Fig. 3. Sources of needle targeting error 
Errors from local field distortions mainly result in errors in the image readout direction. For calibration purposes, minimization of this error can be achieved by acquiring two sets of fiducial images, with the readout direction swapped, and only measuring coordinates in the phase encode direction [10]. Gradient field nonlinearity results in both in-plane geometric errors and slice select errors [11]. These errors are specific to the MR system and are corrected for using image warping algorithms applied by the scanner software after image acquisition. Distortion correction algorithms generally do not correct the slice select errors; furthermore, residual in-plane errors remain due to truncation of the spherical harmonic expansion terms [11].

The propagation error exists because the distance from the registration fiducial arrangement to the prostate is much larger than the distance between the fiducials. This source of error is fixed by the device geometry. The kinematic errors result from imperfections in manufacturing of the device, and deflection of materials. As found in the open air calibration experiment, these errors are relatively small $(\sim 0.3 \mathrm{~mm})$, and are considered insignificant. Before calibration, the total error in targeting a point within the MRI is estimated to be as much as $\sim 10 \mathrm{~mm}$.

A simple calibration method has been applied, whereby the MRI image space in the region of the prostate is registered to the device space with a rigid transformation. Registration was performed using Procrustes analysis. Justification for the effectiveness of this method is based on the observations:

1. The main source of error is gradient field nonlinearity (assumes a good shim over the target volume).

2. The gradient field nonlinearity error is a function of position in the scanner and varies only a small amount over small distances (i.e. over the size of a prostate and the registration fiducial arrangement).

3. The patient and device will always be placed in approximately the same position.

The calibration procedure was performed by fitting the device's manipulator arm with an arrangement of two MR-visible spheres. These spheres were positioned over a region the size of a typical prostate and imaged. A 3D rigid transformation was computed between the positions of the fiducials in the images and their supposed locations from the kinematics solution. Before correction, the maximum error in fiducial position was greater than $5 \mathrm{~mm}$. Calibration reduced this to less than $1 \mathrm{~mm}$. This transformation was then applied to subsequent targeting sessions.

\subsection{Targeting Tests}

Time to Target. An experiment was performed to evaluate the potential advantage of using motors in place of manual actuation. The experiment required a user to align the device to a given virtual target and entry point in open-air at different levels of precision: $0.125 \mathrm{~mm}, 0.250 \mathrm{~mm}, 0.500 \mathrm{~mm}$, and $1.00 \mathrm{~mm}$. Six pairs of target and entry points were targeted at each level of precision, and the order in which the points were targeted was randomized for four users. Locations of the target points were representative of targets in each sextant of the prostate. Table 1 shows the time to successfully target the device (and lock it in position) at various levels of precision. 
Table 1. Time to target at various precision levels

\begin{tabular}{lcccc}
\hline & $\mathbf{0 . 1 2 5} \mathbf{~ m m}$ & $\mathbf{0 . 2 5 0} \mathbf{~ m m}$ & $\mathbf{0 . 5 0 0} \mathbf{~ m m}$ & $\mathbf{1 . 0 0} \mathbf{~ m m}$ \\
\hline \hline Mean Time (s) & 38 & 21 & 15 & 11 \\
\hline Standard Deviation (s) & 11 & 6 & 4 & 2 \\
\hline
\end{tabular}

The results show that targeting was possible in less than $40 \mathrm{~s}$, even at a higher precision level than device's accuracy in open air. Since each therapy session requires insertion of no more than 3 or 4 needles, it is reasoned that motorized positioning could save no more than a few minutes in a procedure that lasts about 2 hours. Thus, we conclude that the addition of motorized trajectory alignment is not justified.

Needle Targeting Accuracy. After calibration, as described in section 2.3, the device was used to deliver needles to targets in a 3T scanner (MR750, GE Healthcare, Milwaukee, WI). The targets consisted of $10 \mathrm{~mm}$ spheres of gelatin, doped with gadolinium (Magnevist, $0.005 \mathrm{mmol} / \mathrm{ml}$ ). Nine such spheres were embedded in a rectangular alginate phantom, as alginate has mechanical properties similar to that of pelvis connective tissue [12]. The spheres were arranged in an approximate grid pattern of size $\sim 65 \mathrm{~mm} \times 65 \mathrm{~mm}$, at a depth of $\sim 85 \mathrm{~mm}$ under the surface of the alginate. This arrangement is representative of typical locations of tumours in a prostate relative to the device. The experimental setup is shown in Figure 5a (needles were inserted with the phantom at the isocenter). Figure 5b shows the needles inserted to their targets (axial slices). Note that this is a concatenation of images from needle insertions into each of the nine spheres. The needles appear as bright dots within the gel spheres.

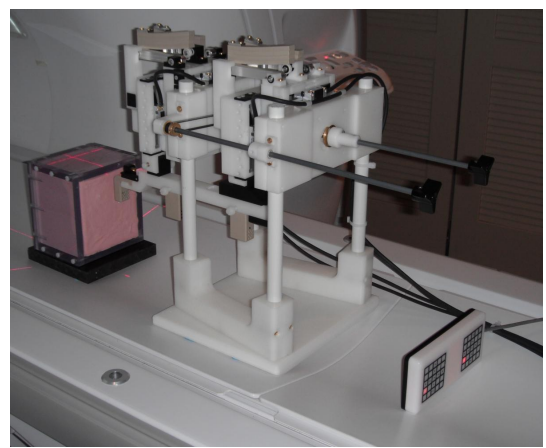

a)

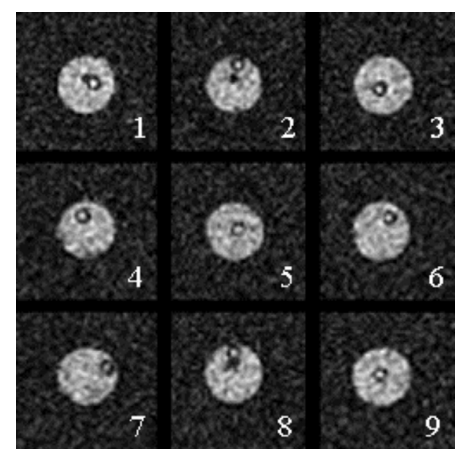

b)

Fig. 5. Needle targeting test: a) setup, b) axial slices showing needles inserted into gel spheres

Positioning error was calculated as the distance between the centroid of the needles after each insertion and the centroid of the gel spheres before needle insertion. The mean error was found to be $2.1+/-1.3 \mathrm{~mm}$. These results show that the device is capable of accurately delivering needles to tumours of the smallest clinically significant size. 


\section{Summary}

We have presented a system for the delivery of MR-guided prostate focal therapies. Targeting tests showed that the device can be aligned to targets in less than 40 seconds with a precision that is greater than its accuracy in open air, validating the use of a manual actuation method. A needle insertion experiment in a gel phantom has shown the device to be capable of delivering needles to targets with an average error of $2.1 \mathrm{~mm}$. Results from initial clinical testing will be presented at the conference.

\section{References}

1. Siegel, R., Naishadham, D., Jemal, A.: Cancer statistics. Cancer J. Clin. 62(1), 10-29 (2012)

2. Klotz, L., Zhang, L., Lam, A., Nam, R., Mamedov, A., Loblaw, A.: Clinical Results of Long-Term Follow-Up of a Large, Active Surveillance Cohort With Localized Prostate Cancer. J. Clin. Oncol. 28(1), 126-131 (2010)

3. Ercole, B., Marietti, S., Fine, J., Albertsen, P.: Outcomes Following Active Surveillance of Men With Localized Prostate Cancer Diagnosed in the Prostate Specific Antigen Era. J. Urology 180(4), 1336-1341 (2008)

4. Haider, M., van der Kwast, T., Tanguay, J., Evans, A., Hashmi, A.-T., Lockwood, G., Trachtenberg, J.: Combined T2-Weighted and Diffusion-Weighted MRI for Localization of Prostate Cancer. Am. J. Roentgenol. 189(2), 323-328 (2007)

5. Schouten, M., Ansems, J., Renema, W.K.J., Bosboom, D., Scheenen, T., Futterer, J.: The accuracy and safety aspects of a novel robotic needle guide manipulator to perform transrectal prostate biopsies. Med. Phys. 37(9), 4744-4750 (2010)

6. Krieger, A., Csoma, C., Iordachita, I., Guion, P., Singh, A., Fichtinger, G., Whitcomb, L.: Design and Preliminary Accuracy Studies of an MRI-Guided Transrectal Prostate Intervention System. In: Ayache, N., Ourselin, S., Maeder, A. (eds.) MICCAI 2007, Part II. LNCS, vol. 4792, pp. 59-67. Springer, Heidelberg (2007)

7. Goldenberg, A., Trachtenberg, J., Yi, Y., Weersink, R., Sussman, M., Haider, M., Ma, L., Kucharczyk, W.: Robot-assisted MRI-guided prostatic interventions. Robotica 28(2), 215-234 (2010)

8. Stoianovici, D., Song, D., Petrisor, D., Ursu, D., Mazilu, D., Mutener, M., Schar, M., Patriciu, A.: "MRI Stealth" robot for prostate interventions. Minimal. Invasiv. Ther. 16(4), 241-248 (2007)

9. Villers, A., McNeal, J., Freiha, F., Stamey, T.: Multiple cancers in the prostate. Morphologic features of clinically recognized versus incidental tumors. Cancer 70(9), 2313-2318 (1992)

10. Haacke, M., Brown, R., Thompson, M., Venkatesan, R.: Magnetic Resonance Imaging: Physical Principles and Sequence Design. J. Wiley-Liss, New York (1999)

11. Wang, D., Strugnell, W., Cowin, G., Doddrell, D., Slaughter, R.: Geometric distortion in clinical MRI systems. Part I: evaluation using a 3D phantom. Magn. Reson. Imaging 22(9), 1211-1221 (2004)

12. Lindner, U., Lawrentschuk, N., Weersink, R., Raz, O., Hlasny, E., Sussman, M., Davidson, S., Gertner, M., Trachtenberg, J.: Construction and Evaluation of an Anatomically Correct Multi-Image Modality Compatible Phantom for Prostate Cancer Focal Ablation. J. Urology 184(1), 352-357 (2010) 